\title{
Weibo Cai (Ed.): Engineering in Translational Medicine
}

\author{
Springer - Verlag London, 2014 ISBN 978-1-4471-4372-7
}

\section{Luigi Mansi}

Published online: 17 July 2014

(C) Springer-Verlag Berlin Heidelberg 2014

This book covers a broad area of engineering research in translational medicine. It is a ponderous publication in two volumes, not available separately, and consists of 999 pages enriched by 267 illustrations, of which 130 are in color. The Editor is Weibo Cai, Associate Professor of Radiology and Medical Physics at the University of Wisconsin - Madison, where he is also Director of the UW Molecular Imaging and Nanotechnology Lab. His international scientific recognition is further supported by his work as Executive Editor of the American Journal of Nuclear Medicine and Molecular Imaging, launched in 2011 and actually fully indexed in PubMed.

Dr. Cai had the capability to coordinate 80 leading experts in preclinical research around the world, coming from academic institutions or other internationally renowned scientific labs. In this way, the most important issues of engineering in translational medicine, together with some less diffuse applications, have been extensively treated by scientists who have a deep knowledge of the present frontiers and of future perspectives in the field.

In 35 focused chapters categorized into five parts, the book treats issues as "Cell and Tissue Engineering" (6 chapters), "Genetic and Protein Engineering" (10 chapters), "Nanoengineering" (10 chapters), "Biomedical Instrumentation" (4 chapters), and "Theranostics and Other Novel Approaches" (4 chapters). Particularly with respect to the biomedical instrumentation (chapters 28-31), there is much to learn about topics such as: Engineering the Next-Generation PET Detectors; Photoacoustic Imaging; Engineering Miniature Imaging Instruments; and Engineering Small Animal Conformal Radiotherapy Systems.
The great value of the publication derives from both its scientific content and from its primacy, being, to my knowledge, the first comprehensive reference book that focuses on "Engineering" and "Translational Medicine".

In this way, it may represent a fundamental instrument to enter into the attractive world of Translational medicine, which bridges basic research to the clinical practice, having as the final goal a new paradigm: personalized molecular medicine centred on the individual patient and not on the disease.

Being that each chapter is written as a commentary on the advances currently being made, the reader may have a general vision of the opportunities and challenges in each field, finding in the extensive bibliography the impulse to learn more about a particular subject.

In conclusion, this book may be considered an invaluable resource at first for scientists who want to have an updated overview of the whole scenario or a reference book to easily find information on the most advanced procedures. Furthermore, this publication may be very useful either for the training of students and residents, who may learn the fundamentals of translational medicine, or for the continuing education of clinicians already working in a medical practice. In this way all of them could open their eyes to a new world, one which is going to revolutionize the pillars of knowledge in biology and medicine. Although the progress in this field is so fast as to require continuous updating in scientific journals, this publication will certainly remain relevant for many years to come.

L. Mansi ( $\bowtie)$

Nuclear Medicine, Second University of Naples, Naples, Italy

e-mail: luigi.mansi@unina2.it 